\title{
Safety and impact on cardiovascular events of long-term multifactorial treatment in patients with metabolic syndrome and abnormal liver function tests: a post hoc analysis of the randomised ATTEMPT study
}

\author{
Vassilios G. Athyros ${ }^{1}$, Olga Giouleme², Emmanouel S. Ganotakis ${ }^{3}$, Moses Elisaf4, \\ Konstantinos Tziomalos ${ }^{3}$, Themistoklis Vassiliadis ${ }^{3}$, Evangelos N. Liberopoulos ${ }^{5}$, Eleni Theocharidou ${ }^{6}$, \\ Asterios Karagiannis ${ }^{1}$, Dimitri P. Mikhailidis ${ }^{5}$; for the Assessing The Treatment Effect in Metabolic \\ Syndrome Without Perceptible diabeTes (ATTEMPT) Collaborative Group
}

\begin{abstract}
12nd Propedeutic Department of Internal Medicine, Medical School, Aristotle University of Thessaloniki, Hippocration Hospital, Thessaloniki, Greece

2Division of Gastroenterology, $1^{\text {st }}$ Propedeutic Department of Internal Medicine, Medical School, Aristotle University of Thessaloniki, Thessaloniki, Greece

${ }^{3}$ Department of Internal Medicine, Medical School, University of Crete, Heraklion, Greece

${ }^{4}$ Department of Internal Medicine, University of Ioannina, Medical School, Ioannina, Greece

${ }^{5}$ Department of Clinical Biochemistry, Royal Free Campus, University College London Medical School, University College London (UCL), United Kingdom

6Liver Transplant, The Royal Free Sheila Sherlock Liver Centre, Royal Free Hospital, Royal Free Hampstead NHS Trust and UCL, London, United Kingdom
\end{abstract}

Submitted: 12 September 2011

Accepted: 12 September 2011

Arch Med Sci 2011; 7, 5: 796-805

DOI: 10.5114 /aoms.2011.25554

Copyright (c) 2011 Termedia \& Banach

\begin{abstract}
Introduction: Non-alcoholic fatty liver disease (NAFLD), a hepatic manifestation of metabolic syndrome (MetS), is common and accounts for $80 \%$ of cases of elevated liver function tests (LFTs). We assessed the long-term effects of multifactorial intervention on LFTs and their association with cardiovascular disease (CVD) events in patients with MetS without diabetes mellitus or CVD. Material and methods: This prospective, randomized, open label study included 1,123 patients (aged 45-65 years). Patients received intensive lifestyle intervention and pharmacotherapy: atorvastatin in all patients (low density lipoprotein cholesterol [LDL-C] targets of $<100 \mathrm{mg} / \mathrm{dl}$ [group A] or $<130 \mathrm{mg} / \mathrm{dl}$ [group B]), inhibitors of the renin-angiotensin-aldosterone axis for hypertension, metformin for dysglycaemia and orlistat for obesity.

Results: Among participants, 326 had modestly elevated LFTs and ultrasonographic (US) evidence of NAFLD (165 patients in group A2 and 161 patients in group B2). The NAFLD resolved during the 42 -month treatment period in $86 \%$ of patients in group A2 and in $74 \%$ of patients in group B2 $(p<0.001)$. In both groups nearly $90 \%$ of patients attained lipid goals. Mean LDL-C and TG levels were higher in group B2 than in group A2 $(p<0.001)$. There were no CVD events in group A2 whereas 5 non-fatal events occurred in group B2 (log-rank$p=0.024)$. There were no major side-effects.

Conclusions: Attaining multiple treatment targets is safe and beneficial in primary prevention patients with MetS and NAFLD. Lipid levels and LFTs normalized, US findings associated with NAFLD resolved and no CVD events occurred in patients with LDL-C levels $<100 \mathrm{mg} / \mathrm{dl}$ (group A2). Resolution of NAFLD might have contributed to the prevention of CVD events.
\end{abstract}

Key words: non-alcoholic fatty liver disease, metabolic syndrome, multifactorial treatment, atorvastatin, cardiovascular disease.

\section{Corresponding author:}

Dimitri P. Mikhailidis BSc, MSC, MD, FRSPH, FCP, FFPM, FRCP, FRCPath

Department of Clinical Biochemistry

Royal Free Hospital Campus University College London Medical School Pond Street, London NW3 2QG, United Kingdom Phone: +44 2078302258 Fax: +44 2078302235 E-mail: mikhailidis@aol.com 


\section{Introduction}

Non-alcoholic fatty liver disease (NAFLD) is a term used for a wide spectrum of hepatic pathology ranging from simple steatosis to non-alcoholic steatohepatitis (NASH) and cirrhosis in individuals who do not consume large amounts of alcohol [1]. There is growing interest in NAFLD because it accounts for approximately $80 \%$ of cases of elevated liver function tests (LFTs) in the American population and it affects approximately $20-25 \%$ of American adults [2-4]. Reports from Japan and Italy suggest a similar prevalence $[5,6]$. In simple steatosis, present in up to $30 \%$ of the general population and in $75 \%$ of obese patients, triglycerides (TGs) accumulate in hepatocytes, occupying more than $5 \%$ of their volume. Simple steatosis has been reported to be associated with increased body mass index (BMI), abnormal glucose tolerance, insulin resistance and other components of the metabolic syndrome (MetS) [1,7]. Accordingly, NAFLD is considered by some as the hepatic manifestation of MetS [8]. In turn, MetS confers an increased risk for cardiovascular disease (CVD) morbidity and mortality $[9,10]$ and all-cause mortality [9], even in the absence of CVD and/or diabetes mellitus (DM) [10, 11]. Patients with NAFLD have $69 \%$ higher all-cause mortality rates than the general population; patients with NASH have an even higher rate [12]. These higher rates are mainly due to CVD events and to a lesser extent due to liver disease-associated mortality [12]. In patients with both NAFLD and MetS the CVD risk appears to be even higher [8].

We recently showed that statin treatment, on top of optimal secondary prevention therapy, is safe and can improve LFTs and reduce CVD morbidity in patients with coronary heart disease with modestly abnormal LFTs, possibly due to NAFLD [13]. However, there are no data on the effect of multifactorial treatment, including a statin, on NAFLD and the related CVD events in primary prevention patients with MetS. This post hoc analysis of the Assessing The Treatment Effect in Metabolic Syndrome Without Perceptible diabeTes (ATTEMPT) study [14] was undertaken to address this issue.

\section{Material and methods}

The study protocol was described in detail in the original paper [14].

\section{Study population}

This is a prospective, randomised, target-based, intention-to-treat, open label study on a selected sample from throughout Greece. All participants had to meet the American Heart Association diagnostic criteria [15] for MetS and attended the participating outpatient clinics, considered to specialise in at least 1 MetS component. Specifically, at least 3 of the following criteria should be present: TG levels > $150 \mathrm{mg} / \mathrm{dl}(1.7 \mathrm{mmol} / \mathrm{l})$; high density lipoprotein cholesterol (HDL-C) levels $<40 \mathrm{mg} / \mathrm{dl}$ $(1.0 \mathrm{mmol} / \mathrm{l})$ for men or $<50 \mathrm{mg} / \mathrm{dl}(1.3 \mathrm{mmol} / \mathrm{l})$ for women; blood pressure (BP) $>130 / 85 \mathrm{mmHg}$ or treatment for the latter 3 conditions; waist circumference $(\mathrm{WC})>102 \mathrm{~cm}$ in men or $>88 \mathrm{~cm}$ in women; and fasting plasma glucose (FPG) $>100 \mathrm{mg} / \mathrm{dl}(5.6 \mathrm{mmol} / \mathrm{l})$ [15].

Exclusion criteria in our study were as follows: TG levels $>500 \mathrm{mg} / \mathrm{dl}(5.6 \mathrm{mmol} / \mathrm{l})$, pregnancy or lactation, hormone replacement therapy, active hepatic disease (known hepatitis or unexplained persistent transaminases elevation > $100 \mathrm{IU} / \mathrm{I}$ ), secondary or resistant hypertension, advanced chronic kidney disease (CKD) (estimated glomerular filtration rate $\left.<30 \mathrm{ml} / \mathrm{min} / 1.73 \mathrm{~m}^{2}\right)$ and the presence of CVD and/or DM (FPG $\geq 126 \mathrm{mg} / \mathrm{dl}$ $(7 \mathrm{mmol} / \mathrm{l})$ and/or glycosylated haemoglobin $\left(\mathrm{HbA}_{1 \mathrm{c}}\right)$ $>6 \%$. The CVD was excluded on the basis of personal history, physical examination and if needed an exercise stress test. Among screened patients, 1,320 agreed to be followed for a longer period (3.5 years) and 1,123 had the appropriate age range (45-65 years old) for CVD risk score estimation and were enrolled in the study and were randomised to the 2 treatment groups using block (of 4) randomization. Randomization was performed by VGA and the rest of the investigators were blinded to treatment.

\section{Study protocol}

The protocol of the ATTEMPT study included multifactorial targeting of all CVD risk factors, including components of the MetS, in all patients during a 42-month period.

Lifestyle advice: All subjects received lifestyle advice. This included exercise (walking for at least 30 min 5 days a week (150 min) or equivalent exercise) and hypolipidaemic (National Cholesterol Education Program Adult Treatment Panel III) hypocaloric diet [15]. At baseline there was an interview with a qualified dietician. Dietary preferences were recorded and a specific 7-day diet sheet was handed to the patient at every visit. Daily calorie intake was set at 500 calories below the needs of the patient according to physical activity.

Treatment of hypertension: Hypertension was treated in a stepwise manner with $5 \mathrm{mg} /$ day (titrated up to $40 \mathrm{mg} /$ day) of quinapril. If this was not enough to reach the treatment target, amlodipine (titrated from 5 to $10 \mathrm{mg} /$ day), and then low-dose hydrochlorothiazide $(12.5 \mathrm{mg} /$ day) were added. If one more antihypertensive drug was required, then central acting antihypertensives, low 
dose selective $\beta$-blockers, and finally any other drug were added to reach the treatment target. The intention was to avoid any metabolic side-effects of antihypertensive drug treatment.

Treatment of obesity: In patients with obesity (BMI $>30 \mathrm{~kg} / \mathrm{m}^{2}$ ) orlistat (up to $360 \mathrm{mg} /$ day) was prescribed throughout the study period.

Treatment of impaired fasting glucose (IFG): The IFG was treated with metformin in patients with FPG $>110 \mathrm{mg} / \mathrm{dl}(6.1 \mathrm{mmol} / \mathrm{l})$. The starting dose was $850 \mathrm{mg} /$ day and was increased to $1,700 \mathrm{mg}$ /day if FPG levels remained $>110 \mathrm{mg} / \mathrm{dl}(6.1 \mathrm{mmol} / \mathrm{l})$.

Treatment of dyslipidaemia: Atorvastatin was administered to all subjects; these were randomly allocated to 2 treatment groups. Group A: LDL-C target $<100 \mathrm{mg} / \mathrm{dl}(n=566)$ and group B: LDL-C target of $<130 \mathrm{mg} / \mathrm{dl}(n=557)$. The atorvastatin dose was titrated from 10 to $80 \mathrm{mg} /$ day.

The study protocol was approved by the local and national ethics committees and written informed consent was obtained from each participant before enrolment. The trial was registered 6 years ago in the official government of USA ClinicalTrials.gov (Trial registration number: ID: NCT00416741).

\section{Investigated parameters}

Data collected from the study subjects included a detailed personal medical history, measurements of the components of the MetS (blood lipids, BP, FPG and WC), as well as other CVD risk factors (sex, age, BMI, smoking status and family history of premature CVD). Blood samples were collected after a $12 \mathrm{~h}$ fast (water allowed) between 8 and $10 \mathrm{am}$, in a sitting position. Total cholesterol, HDL-C and TG levels were determined. The LDL-C was calculated by the Friedewald formula (LDL-C $[\mathrm{mg} / \mathrm{dl}]=\mathrm{TC}[\mathrm{mg} / \mathrm{dl}]-(\mathrm{TG}[\mathrm{mg} / \mathrm{dl}] / 5+\mathrm{HDL}-\mathrm{C}$ $[\mathrm{mg} / \mathrm{dl}])$ ). Serum alanine aminotransferase (ALT), aspartate aminotransferase (AST), $\gamma$-glutamyl transpeptidase $(\gamma-\mathrm{GT})$, alkaline phosphatase (AP), creatinine ( $\mathrm{SCr}$ ), uric acid (SUA) and creatine kinase (CK) were also assessed. Estimated glomerular filtration rate $\left(\mathrm{eGFR} \mathrm{ml} / \mathrm{min} / 1.73 \mathrm{~m}^{2}\right)$ was measured using the Modification of Diet in Renal Disease equation: eGFR $=175 \times(\mathrm{SCr})-1.154$ $\times$ (age) $-0.203 \times(0.742$ if female) [http://www . nkdep.nih.gov/professionals/gfr_calculators/idms con.htm]. Biochemical evaluation was carried out in various laboratories that fulfilled internal and external validity control criteria. All measurements in the same centre were performed by the same lab, using the same methods, and therefore results are comparable. In patients with raised LFTs, abdominal ultrasound (US) was performed, and when US findings were suggestive of NAFLD, the patient was characterized as having NAFLD.

\section{Endpoints}

The primary endpoint of the original study was the reduction in the 10 -year eCVD risk at 6 months compared with baseline (of the interventional part of the study, and not the baseline of the epidemiological part of the study) in the total study population $(n=1,123)$. The secondary endpoints were the eCVD risk reduction during the next 3 years, the proportion of patients having MetS at the $42^{\text {nd }}$ month of treatment and the number of CVD events (unstable angina (UA), myocardial infarction (MI), percutaneous coronary intervention $(\mathrm{PCI})$, coronary artery bypass grafting (CABG), stroke, transient ischaemic attack (TIA) and CVD death) in all participants. In the present post hoc analysis the primary endpoint was the effect of multifactorial treatment on the prevalence of NAFLD in patients with MetS and NAFLD in both treatment groups $(n=326)$ and its relation with actual CVD events during the 42-month followup. The secondary endpoint was the relation of NAFLD with chronic kidney disease (CKD).

\section{Safety}

A clinical and laboratory (ALT, AST, CK, SCr) investigation was performed every 6 months at programmed study visits (there were 10-12 such visits).

\section{Statistical analysis}

Intention-to-treat analysis of all patients randomized to the 2 treatment groups was performed. Continuous variables are presented as mean \pm standard deviation (SD) since all parameters were normally distributed according to the KolmogorovSmirnov test. Qualitative variables are presented as relative frequencies. Clinical and laboratory findings between groups were compared by ANOVA. Unpaired Student's $t$-tests were used for comparisons of parametric data and $\chi^{2}$ tests for comparisons of categorical data between the 2 groups at specific time points. The log-rank test was used to compare CVD event rates in the 2 treatment groups. A two-sided $p<0.05$ was considered significant. All analyses were carried out using the SPSS 19.00 software package (SPSS, Inc., Chicago, IL).

\section{Results}

At baseline, 326 patients had modestly elevated LFTs and US evidence of NAFLD (165 patients in group A2 and 161 patients in group B2).

\section{Effect of treatment on LFTs in patients with US evidence of NAFLD}

There was a substantial reduction in all liver enzyme activities in patients with raised LFTs at 
baseline and US evidence of NAFLD. In group A2 ALT was reduced by $48 \%$ (from $60 \pm 11 \mathrm{IU} / \mathrm{I}$ to $31 \pm 5 \mathrm{IU} / \mathrm{l}$; $p<0.001$ ), AST by $47 \%$ (from $51 \pm 10$ IU/I to $27 \pm 4$ IU/l; $p<0.001$ ), $\gamma$-GT by $60 \%$ (from $91 \pm 15 \mathrm{IU} / \mathrm{l}$ to $36 \pm 6 \mathrm{IU} / \mathrm{l} ; p<0.001$ ) and AP by $43 \%$ (from $171 \pm 28$ $\mathrm{IU} / \mathrm{I}$ to $85 \pm 9 \mathrm{IU} / \mathrm{l} ; p<0.001)$ during the 42 -month follow-up (Table I and Figure 1, panel 1). In group B2 ALT was reduced by $42 \%$ (from $61 \pm 12$ IU/I to $35 \pm 6$ IU/l; $p<0.001$ ), AST by $40 \%$ (from $52 \pm 11 \mathrm{IU} / \mathrm{I}$ to $31 \pm 6 \mathrm{IU} / \mathrm{l} ; p<0.001$ ), $\gamma$-GT by $52 \%$ (from $93 \pm 14 \mathrm{IU} / \mathrm{I}$ to $44 \pm 6 \mathrm{IU} / \mathrm{l} ; p<0.001$ ) and AP by $40 \%$ (from 169 $\pm 32 \mathrm{IU} / \mathrm{I}$ to $101 \pm 15 \mathrm{IU} / \mathrm{I} ; p<0.001)$ during the 42-month follow-up (Table I and Figure 1, panel 2). Reductions in ALT, AST, $\gamma$-GT and AP in group A2 were larger than in group B2 $(p<0.05$ for all 4 comparisons).

At the end of the study, $86 \%(n=142)$ of the patients in group A2 had normal LFTs and no US evidence of NAFLD, $8 \%(n=13)$ had normal LFTs but US evidence of NAFLD and $6 \%(n=10)$ had both elevated LFTs and US evidence of NAFLD. In group B2, at the end of the study $74 \%$ of the patients ( $n=119)$ had normal LFTs and no US evidence of NAFLD, 15\% $(n=24)$ had normal LFTs but some US evidence of NAFLD and $11 \%(n=18)$ had both elevated LFTs and US evidence of NAFLD. NAFLD resolution rate was higher in group $A 2$ than in group B2 (86 vs. $74 \% ; p=0.0012$ ).

Table I. Characteristics of patients with MetS and NAFLD according to treatment allocation (group A2, LDL-C target < 100 mg/dl; group B2, LDL-C target < $130 \mathrm{mg} / \mathrm{dl}$ )

\begin{tabular}{|c|c|c|c|c|c|c|}
\hline \multirow[t]{3}{*}{ Parameter } & \multicolumn{6}{|c|}{ Patients with abnormal LFTs at baseline $(n=326)$} \\
\hline & \multicolumn{3}{|c|}{ Group A2 $(n=165)$} & \multicolumn{3}{|c|}{ Group B2 $(n=161)$} \\
\hline & Baseline & 42 months & Value of $p$ & Baseline & 42 months & Value of $p$ \\
\hline Age [years] & $58 \pm 7$ & - & - & $57 \pm 9$ & - & - \\
\hline Family history of CVD [\%] & 34 & - & - & 36 & - & - \\
\hline Smoking [\%] & 33 & 25 & $<0.001$ & 32 & 25 & $<0.001$ \\
\hline Body mass index $\left[\mathrm{kg} / \mathrm{m}^{2}\right]$ & $33 \pm 4$ & $29 \pm 4$ & $<0.001$ & $32 \pm 5$ & $29 \pm 3$ & $<0.001$ \\
\hline Weight [kg] & $89 \pm 14$ & $82 \pm 11$ & $<0.001$ & $89 \pm 13$ & $83 \pm 12$ & $<0.001$ \\
\hline Systolic blood pressure [mmHg] & $145 \pm 13$ & $125 \pm 9$ & $<0.001$ & $144 \pm 11$ & $124 \pm 10$ & $<0.001$ \\
\hline Diastolic blood pressure [mmHg] & $91 \pm 9$ & $80 \pm 4$ & $<0.001$ & $90 \pm 10$ & $79 \pm 5$ & $<0.001$ \\
\hline $\mathrm{HDL}-\mathrm{C}[\mathrm{mg} / \mathrm{dl}]$ & $45 \pm 11$ & $52 \pm 7$ & $<0.001$ & $46 \pm 10$ & $51 \pm 8$ & $<0.001$ \\
\hline Triglycerides [mg/dl] & $220 \pm 47$ & $123 \pm 20$ & $<0.001$ & $218 \pm 50$ & $142 \pm 34^{*}$ & $<0.001$ \\
\hline Waist circumference [cm] & $105 \pm 9$ & $98 \pm 8$ & $<0.001$ & $104 \pm 11$ & $99 \pm 7$ & $<0.001$ \\
\hline Blood glucose [mg/dl] & $106 \pm 12$ & $98 \pm 9$ & $<0.001$ & $107 \pm 13$ & $97 \pm 8$ & $<0.001$ \\
\hline Total cholesterol [mg/dl] & $255 \pm 44$ & $165 \pm 24$ & $<0.001$ & $256 \pm 41$ & $186 \pm 26^{*}$ & $<0.001$ \\
\hline LDL-C [mg/dl] & $168 \pm 38$ & $92 \pm 12^{*}$ & $<0.001$ & $171 \pm 36$ & $110 \pm 20^{*}$ & $<0.001$ \\
\hline Metabolic syndrome [\%] & 100 & 15 & $<0.001$ & 100 & 16 & $<0.001$ \\
\hline Abdominal obesity [\%] & 90 & 50 & $<0.001$ & 91 & 52 & $<0.001$ \\
\hline Hypertension [\%] & 85 & 11 & $<0.001$ & 84 & 10 & $<0.001$ \\
\hline High TG levels [\%] & 79 & 10 & $<0.001$ & 80 & 12 & $<0.001$ \\
\hline Impaired fasting glucose [\%] & 72 & 9 & $<0.001$ & 71 & 8 & $<0.001$ \\
\hline Low HDL-C [\%] & 61 & 8 & $<0.001$ & 60 & 7 & $<0.001$ \\
\hline ALT [IU/I]) & $60 \pm 11$ & $31 \pm 5$ & $<0.001$ & $61 \pm 12$ & $35 \pm 6^{*}$ & $<0.001$ \\
\hline AST [IU/I] & $51 \pm 10$ & $27 \pm 4$ & $<0.001$ & $52 \pm 11$ & $31 \pm 6^{\star}$ & $<0.001$ \\
\hline$\gamma$-GT [IU/I] & $91 \pm 10$ & $36 \pm 5$ & $<0.001$ & $93 \pm 11$ & $44 \pm 6^{\star}$ & $<0.001$ \\
\hline Alkaline phosphatase [IU/I] & $171 \pm 28$ & $86 \pm 9$ & $<0.001$ & $169 \pm 32$ & $101 \pm 15^{\star}$ & $<0.001$ \\
\hline CVD events & \multicolumn{3}{|c|}{ Expected events: $8-11$} & \multicolumn{3}{|c|}{ Expected events: $10-13$} \\
\hline
\end{tabular}

${ }^{*} p<0.001$ vs. group A2, MetS - metabolic syndrome, NAFLD - non-alcoholic fatty liver disease, LFTs - liver function tests, CVD - cardiovascular disease, $L D L-C$ - low density lipoprotein cholesterol, HDL-C - high density lipoprotein cholesterol, TG - triglycerides, ALT - alanine aminotransferase, AST - aspartate aminotransferase 
LTs in group A2

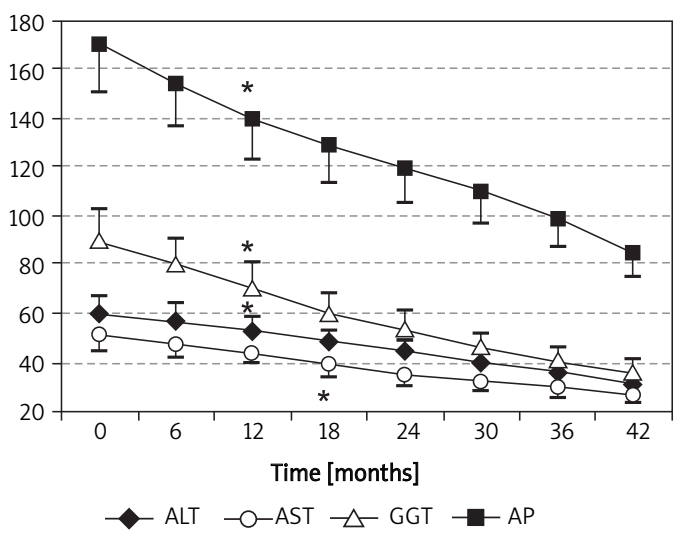

LTs in group B2
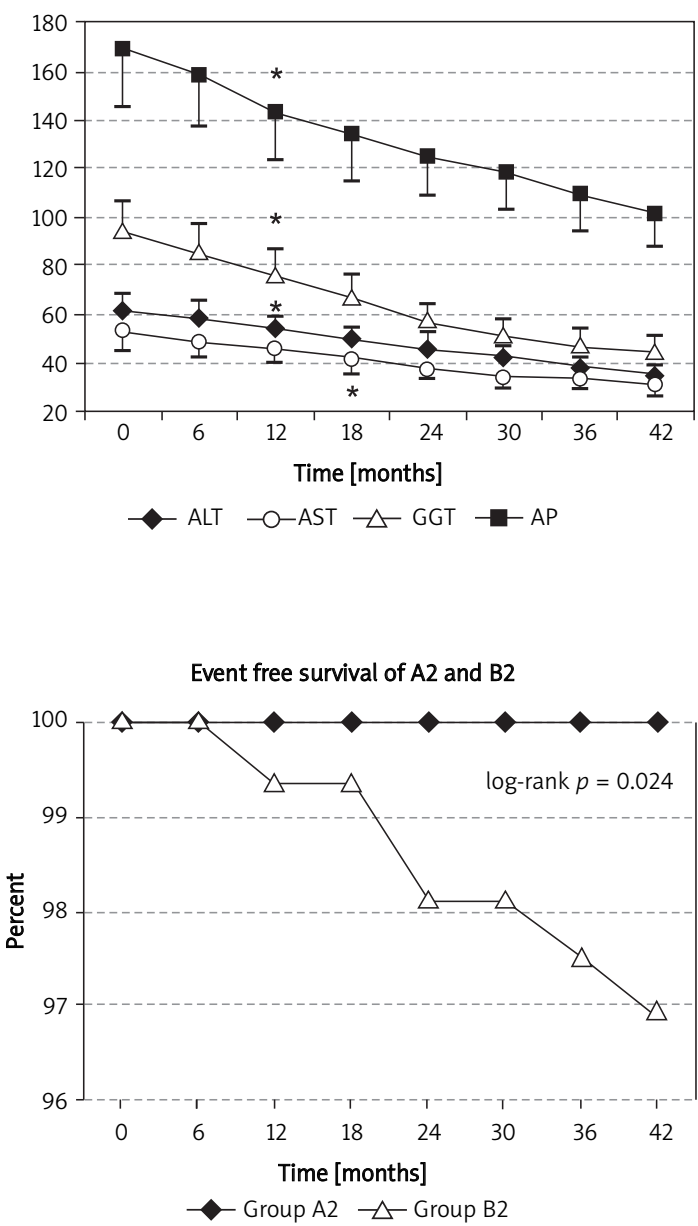

Figure 1. Time course of changes in alanine aminotransferase (ALT), aspartate aminotransferase (AST), $\gamma$-glutamyl transpeptidase $(\gamma$-GT), and alkaline phosphatase (AP) levels (panel 1 for group A2 $(n=165)$ and panel 2 for group B2 $(n=161))$ and event-free survival during the study in groups $\mathrm{A} 2$ and B2 (panel 3)

${ }^{\star} p<0.05$ vs. baseline, group $A 2-$ patients with an LDL-C target $<100 \mathrm{mg} / \mathrm{dl}$, group B2 - patients with an LDL-C target $<130 \mathrm{mg} / \mathrm{dl}$

\section{Effects of treatment on other parameters in patients with NAFLD}

\section{Multifactorial treatment in patients with NAFLD}

Atorvastatin was used in all patients with forced dose titration (10 mg/day to $80 \mathrm{mg} /$ day). In patients with NAFLD, the mean dose of atorvastatin during the study was $34 \mathrm{mg} /$ day and $24 \mathrm{mg} /$ day in group A2 and B2, respectively. The adherence to atorvastatin during the study was high (92\%). The LDL-C and TGs were significantly higher $(p<0.05)$ in group B2 than in group A2 during the study (Table I). Hypertension was treated with quinapril (mean dose $20 \mathrm{mg} /$ day in both groups; $n=229,70 \%$ of patients) and if needed with amlodipine (mean dose $8 \mathrm{mg} /$ day in both groups; $n=131,40 \%$ of patients), and then, if needed, with hydrochlorothiazide (mean dose $12.5 \mathrm{mg} /$ day in both groups; $n=83,25 \%$ of patients). About $28 \%$ of patients were on angiotensin receptor blockers (ARBs), $12 \%$ on centrally acting antihypertensive drugs, $10 \%$ on $\beta$-blockers and $17 \%$ on various other antihypertensive agents. In patients with evidence of NAFLD and $\mathrm{a} B \mathrm{BI}>30 \mathrm{~kg} / \mathrm{m}^{2}$, orlistat was used in a mean dose of $290 \mathrm{mg} /$ day $(n=169)$. Metformin was used in a mean dose of $950 \mathrm{mg} /$ day in 152 patients with IFG.

The use of drugs comprising the multifactorial intervention and the doses administered (with the exception of atorvastatin) did not differ between treatment groups (Table I). Only the atorvastatin doses used and the lipid values achieved differed significantly between the 2 treatment groups.

\section{Changes in measured parameters in patients with NAFLD}

MetS components: The most common MetS component was central obesity, followed by hypertension and high TG and FPG levels and lastly HDL-C. The most prevalent phenotype (19\%) was that with elevated BP (or on antihypertensive treatment), increased WC, high TGs and increased FPG. Baseline LDL-C levels were $170 \mathrm{mg} / \mathrm{dl}$. None of the measured parameters differed significantly between groups A2 and B2 at baseline. At the end of the study, only $10 \%$ of the patients with NAFLD still had MetS (persistent cases), but with fewer components.

Lipid levels: At the $42^{\text {nd }}$ treatment month LDL-C was substantially reduced in both groups ( $92 \pm 12$ $\mathrm{mg} / \mathrm{dl}$ in group A2 vs. $110 \pm 20 \mathrm{mg} / \mathrm{dl}$ in group B2, $p<0.001$ ) (Table I). Approximately $90 \%$ of the study population achieved the allocated LDL-C target ( $<100 \mathrm{mg} / \mathrm{dl}$ in group A2 and $<130 \mathrm{mg} / \mathrm{dl}$ in group B2; Table I). Patients in group A2 had lower TG levels than group B2 $(123 \pm 20 \mathrm{mg} / \mathrm{dl}$ vs. 142 $\pm 34 \mathrm{mg} / \mathrm{dl}, p<0.001)$. The HDL-C levels showed a comparable increase in both groups. 
Renal function: A substantial proportion of patients with NAFLD $(137 / 326,42 \%)$ had stage 3 CKD (eGFR 30-59 ml/min/1.72 $\mathrm{m}^{2}$ ). This proportion was higher than in patients without NAFLD (212/797, 26\%; $p<0.001)$. In patients with NAFLD and stage 3 CKD, eGFR was increased by $13 \%$ vs. baseline $(p<0.001)$.

Other CVD risk factors: The BMI, FPG levels and the prevalence of hypertension were significantly reduced during the 42 months of treatment (Table I). Smaller but significant improvements were observed in WC.

New onset diabetes (NOD): The incidence of NOD during the 42-month follow-up was very low, 0.83$1.00 / 100$ patient-years in patients with MetS and MetS with impaired fasting glucose, respectively.

High sensitivity C-reactive protein ( $h S C R P$ ) levels: The hsCRP levels declined in group A2 more than in group B2 (from $4.0 \mathrm{mg} / \mathrm{l}$ at baseline to $0.7 \mathrm{mg} / \mathrm{l}$ at 42 months in group A2 $(p<0.001)$ and from $4.1 \mathrm{mg} / \mathrm{l}$ to $1.4 \mathrm{mg} / \mathrm{l}$ over the same time period in group B2 $(p<0.001) ; p<0.05$ for the comparison between the 2 groups during the entire study).

\section{The CVD events in NAFLD patients}

During the first 6 months there were no CVD events among the 326 participants with NAFLD. Between the $6^{\text {th }}$ and the $42^{\text {nd }}$ month there was no CVD event in group A2 (Table I and Figure 1, panel 3) whereas 5 non-fatal CVD events (3 nonfatal MIs and 2 PCIs) occurred in group B2 $(p=0.0024$, Table I).

\section{Effect of treatment on LFTs in patients without US evidence of NAFLD}

At baseline 42 patients, apart from the 326 with NAFLD, had elevated LFTs without US confirmation of NAFLD. These had ALT and AST values similar to those of the 326 NAFLD patients but lower values of $\gamma$-GT and AP $(65 \pm 14$ IU/I and $132 \pm 13$ IU/I vs. $92 \pm 12$ and $170 \pm 25 \mathrm{IU} / \mathrm{l}$, respectively; $p<0.05)$. The reduction in both ALT and AST during the study was lower than that achieved in the 326 NAFLD patients ( 26 vs. $45 \%$ in ALT and 24 vs. $43 \%$ in AST values ( $p<0.05$ in comparison to the reduction of ALT and AST values in the 326 NAFLD patients)).

\section{Discussion}

We recently reported that statin treatment is safe and can improve LFTs and reduce CVD morbidity and mortality in secondary prevention patients with modestly abnormal LFTs that were attributable to NAFLD [13]. To our knowledge, the present study is the first prospective, randomized, intention-to-treat, controlled trial to assess the effects of multifactorial intervention on LFTs and related CVD events in primary prevention patients with NAFLD. The key findings are that this multifactorial intervention results in an improvement of LFTs and reduces the CVD event rate. These findings could be attributed to intensive atorvastatin treatment and lower lipid levels, because reductions in LFTs and CVD events in group A2 (with an LDL-C $<100 \mathrm{mg}$ target) were superior to those in the less aggressive group B2 (with an LDL-C target $<130 \mathrm{mg} / \mathrm{dl}$ ), while all other risk factors and interventions were similar in these 2 groups.

Visceral obesity, impaired glucose metabolism, hypertension and dyslipidaemia are prevalent in NAFLD, and this might play a role in the increased CVD risk in NAFLD patients [16]. However, it appears that NAFLD per se might be independently related to increased CVD risk [17-19]. In NAFLD, insulin resistance is related to elevated levels of markers of inflammation, such as CRP and fibrinogen [17]. Moreover, in obesity/NAFLD visceral adipocytes are a major source of the increase in circulating proinflammatory, prothrombotic and proatherogenic factors, collectively termed adipokines, which include tumour necrosis factor- $\alpha$, leptin, plasminogen activator inhibitor-1, interleukin- 6 and angiotensinogen, whereas levels of adiponectin, which has antidiabetic, anti-atherogenic and antiinflammatory properties, are substantially reduced $[17,18]$. Thus, increased pro-atherogenic factors and decreased plasma levels of adiponectin may represent another possible mechanism linking NAFLD and CVD [17-19]. Most of these factors appear to normalize during statin treatment [20].

Given the multifactorial pathogenesis of NAFLD, numerous treatments for various contributory risk factors have been proposed in the past, but there is no single established intervention to be recommended for all cases irrespective of the primary NAFLD risk factor [21]. Weight loss and increased physical activity constitute the cornerstone of NAFLD treatment [21]; however, the compliance of patients to long-term lifestyle modifications is low and therefore pharmacotherapy is frequently necessary [22, 23]. Even modest weight loss (7-10\% of body weight) in overweight patients with NAFLD has been shown to improve LFTs [24]. In addition, improvement or resolution of obesity and MetS has been associated with major improvements in lobular steatosis as well as in liver necroinflammation and fibrosis [24, 25]. In ATTEMPT, intensive lifestyle measures resulted in a $7.3 \%$ weight loss in NAFLD patients (Table I). The use of orlistat is a safe adjunct to intensive lifestyle measures for weight loss [26]. Orlistat, either monotherapy or in combination therapy, also appears to be beneficial in NAFLD [26-30]. In the present study, significant reductions were observed in body weight, BMI and WC, and there is evidence that weight loss induced by orlistat might reverse 
fatty infiltration of the liver and improve insulin resistance and even hepatic fibrosis in obese patients with NAFLD [31]. Thus, the use of orlistat in $51 \%$ of our patients might have contributed to the resolution of NAFLD.

It has also been shown that both metformin and dietary treatment alone ameliorate liver steatosis and metabolic abnormalities in patients with NAFLD [32]. However, metformin plus diet was more effective than dietary treatment alone in normalizing several metabolic parameters in these patients [32]. In contrast to thiazolidinediones, metformin has been shown to improve biochemical markers in NAFLD patients without inducing weight gain [33-35]. In addition, an improvement in body composition and fat distribution as well as a reduction in liver necroinflammation was observed in patients treated with metformin [22]. Thus, the use of metformin by $46 \%$ of our patients might have also played a role in the resolution of NAFLD.

Hypertension appears to be associated with NAFLD [36], and in the ATTEMPT study, hypertension was the most prevalent MetS component [14]. The renin-angiotensin-aldosterone system (RAAS) plays a role in increasing insulin resistance whereas RAAS suppression improves intracellular insulin signalling through activation of PPAR- $\gamma$ receptors [37-39]. This effect attenuates hepatic inflammation and fibrogenesis, which translate into clinical and histological improvement in NAFLD [37-39]. Thus, the fact that $70 \%$ of our patients were on an ACE-I and $28 \%$ on an ARB might have contributed to the improvement in LFTs [36].

Nevertheless, all the above treatments, except for atorvastatin, were equally administered to both treatment groups. Therefore, we mainly attribute the greater reductions in LFT values, higher rates of NAFLD resolution and lower CVD incidence in group A2 compared with group B2 to more aggressive treatment with atorvastatin. The pleiotropic effects of statins, which also appear to be dose related, might have also played a role in the improved outcome of patients who received higher atorvastatin doses $[16,40]$.

We have previously shown that atorvastatin alone or in combination with fenofibrate normalizes LFTs and US findings in patients with NAFLD, free of DM and CVD at baseline [41]. These effects were independently related to statin treatment as well as to reductions in WC, body weight, systolic BP and serum hsCRP, TG, LDL-C, total cholesterol and FPG levels [41]. Other studies have also shown that statins are safe and effective in patients with elevated LFTs because of NAFLD [42, 43]. In a study with histopathological follow-up, NAFLD patients who were on statins exhibited significant reductions in liver steatosis as opposed to patients who were not taking statins [43]. Recently, we showed in patients with coronary heart disease that, besides the resolution of LFTs and improvement in US findings, statin treatment reduced CVD events more in patients with NAFLD than in those without NAFLD (68\% vs. $39 \%$, respectively; $p=0.007$ ) $[13,44]$. The present post hoc analysis of the ATTEMPT study suggests, for the first time in primary prevention, that a higher atorvastatin dose (34 mg/day), which achieved lower LDL-C and TG levels, reduced LFTs values and CVD events more than a lower dose of atorvastatin ( $24 \mathrm{mg} /$ day). This suggests that to achieve maximum benefit, more aggressive LDL-C targets should be pursued for dyslipidaemia associated with MetS-related NAFLD. In ATTEMPT, the differences in LDL-C and TG levels between groups $A 2$ and $B 2$ were 18 and $19 \mathrm{mg} / \mathrm{dl}$, respectively (Table I), whereas there was no significant difference in HDL-C levels between the 2 treatment groups. There are no survival studies that included patients with MetS and NAFLD. However, our findings concur with the effects of atorvastatin on lipids and on CVD incidence in a post hoc analysis of the Treating to New Targets (TNT) trial in patients with MetS [45]. In patients with CVD and MetS included in TNT, the mean on-treatment LDL-C concentrations at 3 months were $99 \mathrm{mg} / \mathrm{dl}$ with atorvastatin $10 \mathrm{mg} /$ day and $72 \mathrm{mg} / \mathrm{dl}$ with atorvastatin $80 \mathrm{mg} /$ day (a difference of $27 \mathrm{mg} / \mathrm{dl} ; p<0.001)$, TGs were $148 \mathrm{mg} / \mathrm{dl}$ and $176 \mathrm{mg} / \mathrm{dl}$ respectively (a difference of $28 \mathrm{mg} / \mathrm{dl}$; $p<0.001)$ and there was no significant difference in HDL-C levels between the 2 treatment groups [45]. At a median follow-up of 4.9 years, major CVD events occurred in $13 \%$ of the patients receiving atorvastatin $10 \mathrm{mg} /$ day and in $9.5 \%$ of the patients receiving atorvastatin $80 \mathrm{mg} /$ day (29\% reduction, $p<0.001$ ) [45]. Similar findings were observed in MetS patients without DM [45]. A subsequent subgroup analysis of the Scandinavian Simvastatin Survival Study showed that patients with MetS benefited from simvastatin therapy by at least as much as those without MetS, despite the relatively high baseline $(190 \mathrm{mg} / \mathrm{dl})$ and on-study $(120 \mathrm{mg} / \mathrm{dl})$ values of LDL-C [46].

Another possible explanation for NAFLD resolution and additional CVD clinical benefit induced by atorvastatin is that liver TG increase is linked with decreased expression of PPAR- $\alpha$, which is prevented by atorvastatin treatment [47]. The increase in PPAR- $\alpha$ expression caused by atorvastatin is associated with reduced liver TGs and plasma non-esterified fatty acid levels, because of increased hepatic fatty acid $\beta$-oxidation activity [47]. Moreover, atorvastatin can ameliorate the very low density lipoprotein (VLDL) apolipoprotein B overproduction state observed in a fructose-fed, insulin-resistant hamster model, and may also 
improve hepatic insulin sensitivity [48]. In addition, MetS-induced abnormalities in plasma markers of inflammation and haemostasis and in monocyte secretory functions are ameliorated by atorvastatin [49]. Indeed, in ATTEMPT a substantial fall in hsCRP levels was recorded. Finally, atorvastatin substantially reduced SUA levels, which are also implicated in NAFLD-related CVD risk [50].

We also observed a significant association between NAFLD and the risk of CKD, 2 independent CVD risk factors [51], which share many important cardio-metabolic risk factors [52]. The prevalence of stage 3 CKD among NAFLD patients was higher than in those without NAFLD ( $42 \%$ vs. $26 \%$; $p<0.001$ ), suggesting a common pathophysiology [52, 53]. Inter-related risk factors, dyslipidaemia and proinflammatory/procoagulant mediators from the fatty/inflamed liver, might play a role in the natural history of obesity-related kidney and liver disease $[52,53]$. The coexistence of these 2 risk factors appears to further increase the CVD risk $[19,51]$. We showed in another post hoc analysis of ATTEMPT that multifactorial treatment improves renal function and lowers SUA levels in patients with stage 3 CKD [54]. This is also true for patients with NAFLD and CKD (a 13\% increase in eGFR was observed) and might have acted in conjunction with NAFLD resolution to minimize CVD events in patients with MetS, NAFLD and CKD. The fact that the incidence of NOD during the 42-month follow-up was very low (0.83-1.00/100 patient-years) in patients with MetS and MetS with impaired fasting glucose, respectively, might have contributed to the low incidence of CVD in our patients [55].

Our findings suggest that atorvastatin therapy might prevent hepatocellular damage [56]. It was shown that the combination of atorvastatin with a standard diet yields greater improvements in $\mathrm{NASH}$ [56]. Thus, atorvastatin therapy not only decreases the levels of cholesterol and TGs, but might also induce a reduction of liver steatosis, inflammation and hepatocellular damage, which will translate to reduction of LFT values [56].

It is possible that most of the 42 patients who had elevated LFTs without US findings of NAFLD had indeed a "milder" form of NAFLD (with less fat deposition). These patients had significant but smaller reductions in ALT and AST than those with both LFT elevations and US findings of NAFLD $(n=326)$. One possible explanation is that multifactorial treatment significantly reduced the higher liver fat content in the latter patients, leading to a greater reduction of LFTs.

This study has several limitations. This is a post hoc analysis of the original survival study with MetS patients having NAFLD. We did not perform biopsies to diagnose NAFLD or its resolution after treatment.
Biopsy is the "gold standard" for diagnosis of NAFLD; hence we tried to strengthen our diagnostic criteria in the light of ethical restrictions associated with liver biopsies (especially if this would be required before and after treatment in a large number of patients). This is why we included both features, LFTs and US. The number of patients analysed is relatively small. Generalisability of study findings will be reinforced by prospective data of larger studies.

In conclusion, within a multifactorial treatment, it is safe to prescribe statins in primary CVD prevention patients with MetS and NAFLD with modestly elevated LFTs. The LFTs are gradually reduced and US findings improve during long-term statin treatment. Moreover, there is a substantial reduction in CVD events when lipid targets are attained. These findings support our earlier suggestion for statin use in NAFLD treatment [57]. Given the high prevalence of NAFLD and its role as a CVD risk factor, properly designed, prospective studies are needed to confirm these findings and allow the incorporation of statin treatment as a major component of NAFLD therapy.

\section{Acknowledgments}

This study was conducted under the auspices of the Hellenic Atherosclerosis Society and was sponsored by Pfizer Hellas through an unrestricted research grant.

\section{Thirty collaborators}

We thank the following physicians for providing patient data: Alexandrides T, Anagnostis P, Bilianou E, Basdekas S, Baharoudis G, Boulokos V, Daskalopoulos G, Giannoglou G, Gossios TD, Griva T, Filippatos TD, Florentin M, Gazi IF, Kakafika A, Kapnia E, Kargiotis K, Katsiki N, Kolovou G, Kostapanos MS, Liopirakis A, Lioudaki E, Michailidou K, Mitsiou E, Nicolaou V, Pagourelias ED, Paletas K, Pappas S, Petridis D, Saougos V, Tolias D.

\section{References}

1. Angulo P. GI epidemiology: nonalcoholic fatty liver disease. Aliment Pharmacol Ther 2007; 25: 883-9.

2. Fatani S, Itua I, Clark P, Wong C, Naderali EK. The effects of diet-induced obesity on hepatocyte insulin signaling pathways and induction of non-alcoholic liver damage. Int J Gen Med 2011; 4: 211-9.

3. Bhala N, Angulo P, van der Poorten D, et al. The natural history of nonalcoholic fatty liver disease with advanced fibrosis or cirrhosis: an international collaborative study. Hepatology 2011 Jun 17. doi: 10.1002/hep.24491. [Epub ahead of print].

4. Clark JM, Brancati FL, Diehl AM. Nonalcoholic fatty liver disease. Gastroenterology 2002; 122: 1649-57.

5. Nomura H, Kashiwagi S, Hayashi J, Kajiyama W, Tani S, Goto M. Prevalence of fatty liver in a general population of Okinawa, Japan. Jpn J Med 1988; 27: 142-9. 
6. Bellentani S, Tiribelli C, Saccoccio G, et al. Prevalence of chronic liver disease in the general population of northern Italy: the Dionysos Study. Hepatology 1994; 20: 1442-9.

7. Bedogni G, Miglioli L, Masutti F, Tiribelli C, Marchesini G, Bellentani S. Prevalence of and risk factors for nonalcoholic fatty liver disease: the Dionysos nutrition and liver study. Hepatology 2005; 42: 44-52.

8. Kim CH, Younossi ZM. Nonalcoholic fatty liver disease: a manifestation of the metabolic syndrome. Cleve Clin J Med 2008; 75: 721-8.

9. Isomaa B, Almgren P, Tuomi T, et al. Cardiovascular morbidity and mortality associated with the metabolic syndrome. Diabetes Care 2001; 24: 683-9.

10. Lakka HM, Laaksonen DE, Lakka TA, et al. The metabolic syndrome and total and cardiovascular disease mortality in middle-aged men. JAMA 2002; 288: 2709-16.

11. Trevisan M, Liu J, Bahsas FB, Menotti A. Syndrome $X$ and mortality: a population-based study. Am J Epidemiol 1998; 148: 958-66.

12. Söderberg C, Stål P, Askling J, et al. Decreased survival of subjects with elevated liver function tests during a 28-year follow-up. Hepatology 2010; 51: 595-602.

13. Athyros VG, Tziomalos K, Gossios TD, et al.; GREACE Study Collaborative Group. Safety and efficacy of long-term statin treatment for cardiovascular events in patients with coronary heart disease and abnormal liver tests in the Greek Atorvastatin and Coronary Heart Disease Evaluation (GREACE) Study: a post-hoc analysis. Lancet 2010; 376: 1916-22.

14. Athyros VG, Ganotakis E, Kolovou G, et al.; for the Assessing The Treatment Effect in Metabolic Syndrome Without Perceptible diabeTes (ATTEMPT) Collaborative Group. Assessing The Treatment Effect in Metabolic syndrome without Perceptible diabeTes (ATTEMPT). A prospective-randomized study in middle aged men and women. Curr Vasc Pharmacol 2011 (in press PMID: 21476961).

15. Grundy SM, Cleeman JI, Daniels SR, et al.; American Heart Association; National Heart, Lung, and Blood Institute. Diagnosis and management of the metabolic syndrome: an American Heart Association/National Heart, Lung, and Blood Institute Scientific Statement. Circulation 2005; 112: 2735-52.

16. Athyros VG, Kakafika AI, Karagiannis A, Mikhailidis DP. Do we need to consider inflammatory markers when we treat atherosclerotic disease? Atherosclerosis 2008; 200: 1-12.

17. Jarrar MH, Baranova A, Collantes R, et al. Adipokines and cytokines in non-alcoholic fatty liver disease. Aliment Pharmacol Ther 2008; 27: 412-21.

18. Estep M, Abawi M, Jarrar M, et al. Association of obestatin ghrelin, and inflammatory cytokines in obese patients with non-alcoholic fatty liver disease. Obes Surg 2011 Jul 9. DOI: 10.1007/s11695-011-0475-1 [Epub ahead of print]

19. Targher G, Bertolini L, Poli F, et al. Nonalcoholic fatty liver disease and risk of future cardiovascular events among type 2 diabetic patients. Diabetes 2005; 54: 3541-6.

20. Athyros VG, Kakafika Al, Tziomalos K, Karagiannis A, Mikhailidis DP. Pleiotropic effects of statins-clinical evidence. Curr Pharm Des 2009; 15: 479-89.

21. Tilg $H$, Moschen A. Weight loss: cornerstone in the treatment of non-alcoholic fatty liver disease. Minerva Gastroenterol Dietol 2010; 56: 159-67.

22. Duvnjak M, Tomasic V, Gomercic M, Smircic Duvnjak L, Barsic N, Lerotic I. Therapy of nonalcoholic fatty liver disease: current status. J Physiol Pharmacol 2009; 60 Suppl 7: 57-66
23. Comar KM, Sterling RK. Drug therapy for non-alcoholic fatty liver disease. Aliment Pharmacol Ther 2006; 23: 207-15.

24. Hickman IJ, Jonsson JR, Prins JB, et al. Modest weight loss and physical activity in overweight patients with chronic liver disease results in sustained improvements in alanine aminotransferase, fasting insulin, and quality of life. Gut 2004; 53: 413-9.

25. Dixon JB, Bhathal PS, Hughes NR, O'Brien PE. Nonalcoholic fatty liver disease: improvement in liver histological analysis with weight loss. Hepatology 2004; 39: 1647-54.

26. Filippatos TD, Derdemezis CS, Gazi IF, Nakou ES, Mikhailidis DP, Elisaf MS. Orlistat-associated adverse effects and drug interactions: a critical review. Drug Saf 2008; 31: 53-65.

27. Assy N, Hussein O, Abassi Z. Weight loss induced by orlistat reverses fatty infiltration and improves hepatic fibrosis in obese patients with non-alcoholic steatohepatitis. Gut 2007; 56: 443-4.

28. Harrison SA, Fecht W, Brunt EM, Neuschwander-Tetri BA. Orlistat for overweight subjects with nonalcoholic steatohepatitis: a randomized, prospective trial. Hepatology 2009; 49: 80-6.

29. Filippatos TD, Kiortsis DN, Liberopoulos EN, Georgoula M, Mikhailidis DP, Elisaf MS. Effect of orlistat, micronised fenofibrate and their combination on metabolic parameters in overweight and obese patients with the metabolic syndrome: the FenOrli study. Curr Med Res Opin 2005; 21: 1997-2006.

30. Filippatos TD, Gazi IF, Liberopoulos EN, et al. The effect of orlistat and fenofibrate, alone or in combination, on small dense LDL and lipoprotein-associated phospholipase A2 in obese patients with metabolic syndrome. Atherosclerosis 2007; 193: 428-37.

31. Ratziu V, Zelber-Sagi S. Pharmacologic therapy of nonalcoholic steatohepatitis. Clin Liver Dis 2009; 13: 667-88.

32. Garinis GA, Fruci B, Mazza A, et al. Metformin versus dietary treatment in nonalcoholic hepatic steatosis: a randomized study. Int J Obes (Lond) 2010; 34: 1255-64.

33. Nair S, Diehl AM, Wiseman M, Farr GH Jr, Perrillo RP. Metformin in the treatment of non-alcoholic steatohepatitis: a pilot open label trial. Aliment Pharmacol Ther 2004; 20: 23-8.

34. Ratziu V, Caldwell S, Neuschwander-Tetri BA. Therapeutic trials in non-alcoholic steatohepatitis: insulin sensitizers and related methodological issues. Hepatology 2010; 52: 2206-15.

35. Caldwell SH, Argo CK, Al-Osaimi AM. Therapy of NAFLD: insulin sensitizing agents. J Clin Gastroenterol 2006; 40 (Suppl 1): S61-6.

36. Donati G, Stagni B, Piscaglia F, et al. Increased prevalence of fatty liver in arterial hypertensive patients with normal liver enzymes: role of insulin resistance. Gut 2004; 53: 1020-3.

37. Georgescu EF. Angiotensin receptor blockers in the treatment of NASH/NAFLD: could they be a first-class option? Adv Ther 2008; 25: 1141-74.

38. Yokohama S, Yoneda M, Haneda M, et al. Therapeutic efficacy of an angiotensin II receptor antagonist in patients with nonalcoholic steatohepatitis. Hepatology 2004; 40: 1222-5.

39. Georgescu EF, Ionescu R, Niculescu M, Mogoanta L, Vancica L. Angiotensin-receptor blockers as therapy for mild-to-moderate hypertension-associated non-alcoholic steatohepatitis. World J Gastroenterol 2009; 15: 942-54.

40. Gibson CM, Pride YB, Hochberg CP, Sloan S, Sabatine MS, Cannon CP; TIMI Study Group. Effect of intensive statin 
therapy on clinical outcomes among patients undergoing percutaneous coronary intervention for acute coronary syndrome. PCI-PROVE IT: A PROVE IT-TIMI 22 (Pravastatin or Atorvastatin Evaluation and Infection TherapyThrombolysis In Myocardial Infarction 22) Substudy. J Am Coll Cardiol 2009; 54: 2290-5.

41. Athyros VG, Mikhailidis DP, Didangelos TP, et al. Effect of multifactorial treatment on non-alcoholic fatty liver disease in metabolic syndrome: a randomised study. Curr Med Res Opin 2006; 22: 873-83.

42. Liberopoulos EN, Athyros VG, Elisaf MS, Mikhailidis DP. Statins for non-alcoholic fatty liver disease: a new indication? Aliment Pharmacol Ther 2006; 24: 698-9.

43. Ekstedt M, Franzén LE, Mathiesen UL, Holmqvist $M$, Bodemar G, Kechagias S. Statins in non-alcoholic fatty liver disease and chronically elevated liver enzymes: a histopathological follow-up study. J Hepatol 2007; 47: 135-41.

44. Athyros VG, Tziomalos K, Daskalopoulos GN, Karagiannis A, Mikhailidis DP. Statin-based treatment for cardiovascular risk and non-alcoholic fatty liver disease. Killing two birds with one stone? Ann Med 2011; 43: 167-71.

45. Deedwania P, Barter P, Carmena R, et al.; the Treating to New Targets Investigators. Reduction of low-density lipoprotein cholesterol in patients with coronary heart disease and metabolic syndrome: analysis of the Treating to New Targets study. Lancet 2006; 368: 919-28.

46. Pyorala K, Ballantyne CM, Gumbiner B, et al. Reduction of cardiovascular events by simvastatin in nondiabetic coronary heart disease patients with and without the metabolic syndrome: subgroup analyses of the Scandinavian Simvastatin Survival Study (4S). Diabetes Care 2004; 27: 1735-40.

47. Roglans N, Sanguino E, Peris C, et al. Atorvastatin treatment induced peroxisome proliferator-activated receptor alpha expression and decreased plasma nonesterified fatty acids and liver triglyceride in fructosefed rats. J Pharmacol Exp Ther 2002; 302: 232-9.

48. Mangaloglu L, Cheung RC, Van Iderstine SC, Taghibiglou C, Pontrelli L, Adeli K. Treatment with atorvastatin ameliorates hepatic very-low-density lipoprotein overproduction in an animal model of insulin resistance, the fructose-fed Syrian golden hamster: evidence that reduced hypertriglyceridemia is accompanied by improved hepatic insulin sensitivity. Metabolism 2002; 51: 409-18.

49. Krysiak R, Gdula-Dymek A, Bachowski R, Okopien B. Pleiotropic effects of atorvastatin and fenofibrate in metabolic syndrome and different types of pre-diabetes. Diabetes Care 2010; 33: 2266-70.

50. Katsiki N, Athyros VG, Karagiannis A, Mikhailidis DP. Hyperuricaemia and non-alcoholic fatty liver disease (NAFLD): a relationship with implications for vascular risk? Curr Vasc Pharmacol 2011 Mar 10 [Epub ahead of print. PMID: 21388346].

51. Targher G, Chonchol M, Pichiri I, Zoppini G. Risk of cardiovascular disease and chronic kidney disease in diabetic patients with non-alcoholic fatty liver disease: just a coincidence? J Endocrinol Invest 2011 Mar 21 [Epub ahead of print. PMID: 21427524].

52. Targher G, Chonchol M, Zoppini G, Abaterusso C, Bonora E. Risk of chronic kidney disease in patients with nonalcoholic fatty liver disease: is there a link? J Hepatol 2011; 54: 1020-9.

53. Catalano D, Trovato GM, Martines GF, Pirri C, Trovato FM. Renal function and severity of bright liver. Relationship with insulin resistance, intrarenal resistive index, and glomerular filtration rate. Hepatol Int 2011 Jan 28 [Epub ahead of print. PMID: 21484130].
54. Athyros VG, Karagiannis A, Ganotakis ES, et al.; the ATTEMPT Collaborative Group. Improvement of renal function and serum uric acid levels by multifactorial intervention and their effects in clinical outcome in patients with multiple cardiovascular disease risk factors. A post hoc analysis of the ATTEMPT study. Curr Med Res Opinion 2011 doi:10.1185/03007995.2011.595782.

55. Athyros VG, Elisaf M, Alexandrides T, et al.; the ATTEMPT Collaborative Group. Long-term impact of multifactorial treatment on new-onset diabetes and related cardiovascular events in metabolic syndrome. A post hoc ATTEMPT analysis. Angiology 2011, DOI: 10.1177/ 0003319711421341.

56. Martín-Castillo A, Castells MT, Adánez G, Polo MT, Pérez BG, Ayala I. Effect of atorvastatin and diet on nonalcoholic fatty liver disease activity score in hyperlipidemic chickens. Biomed Pharmacother 2010; 64: 275-81.

57. Liberopoulos EN, Athyros VG, Elisaf MS, Mikhailidis DP. Statins for non-alcoholic fatty liver disease: a new indication? Aliment Pharmacol Ther 2006; 24: 698-9. 